Kreisky, Eva/Sauer, Birgit (Hg.) (1997a): Das geheime Glossar der Politikwissenschaft. Geschlechtskritische Inspektion der Kategorien einer Disziplin, Frankfurt a.M./New York

Kreisky, Eva/Sauer, Birgit (1997b): Heimlichkeit und Kanonisierung. Einführende Bemerkungen zur Begriffsbildung in der Politikwissenschaft, in: Kreisky, Eva/Sauer, Birgit (Hg.), Das geheime Glossar der Politikwissenschaft. Geschlechtskritische Inspektion der Kategorien einer Disziplin, Frankfurt a.M./New York, S. 7-45

Leischko, Harriet (1993): Die ersten österreichischen Nationalökonominnen, Diplomarbeit an der Wirtschaftsuniversität Wien, Wien

Nautz, Jürgen (1997): Zwischen Emanzipation und Integration. Die Frauen der Wiener Schule für Nationalökonomie, in: Fischer, Lisa/Brix, Emil (Hg.), Die Frauen der Wiener Moderne, Wien/München, S. 64-82

Pujol, Michèle A. (1992): Feminism and Anti-Feminism in Early Economic Thought, Aldershot: Edward Elgar

Rudolph, Hedwig (1990): Der männliche Blick in der Nationalökonomie, in: Hausen, Karin/ Nowotny, Helga (Hg.), Wie männlich ist die Wissenschaft?, Frankfurt a. M., S. I29-I44 Weiland, Daniela (1983): Geschichte der Frauenemanzipation in Deutschland und Österreich, Hermes Handlexikon, Düsseldorf

\title{
Finanzspekulation, Arbeitslosigkeit und Staatsverschuldung Financial Speculation, Unemployment and Public Debt Stephan Schulmeister*
}

Gewinnstreben und Vermögensbildung von Unternehmen und Haushalten haben sich in den vergangenen 25 Jahren zunehmend auf Finanzveranlagung und -spekulation konzentriert. Dieser »Megatrend « ist in Deutschland besonders markant ausgeprägt. »Paradekonzerne« wie Siemens stoßen immer mehr Teile ihrer industriellen Produktion ab und weiten gleichzeitig ihre "treasuries« aus. Diese erzielen auf den Finanzmärkten hohe Renditen. Solche »opportunity profits« beschränken das Volumen realwirtschaftlicher Investitionen, da realwirtschaftlich Anlegende ähnlich hohe Renditen wie auf den Finanzmärkten fordern. Die Banken verlagern (daher) ihre Aktivitäten von der Investitionsfinanzierung zum "asset management" (Musterbeispiel: Deutsche Bank).

* Österreichisches Institut für Wirtschaftsforschung, Wien. Ich danke Eva Sokoll für die statistische Assistenz sowie Karl Aiginger, Thomas Leoni, Angela Köppl, Ina Meyer, Kurt Rothschild, Margit Schratzenstaller, Gunther Tichy und Ewald Walterskirchen für Anregungen und Kritik. 
Der Finanzboom hat auch die »Renditeansprüchlichkeit« der privaten Haushalte gesteigert. Unterschiedlichste Investmentfonds verheißen entsprechende Renditen. Bei nahezu stagnierender Realwirtschaft stellen Bewertungsgewinne die wichtigste Profitquelle dar: Hohe Renditen werden daher nur in Phasen boomender Aktienkurse erzielt.

Große Privatvermögen werden vorzugsweise in Hedge Funds vermehrt. Diese erzielen ihre Profite primär auf zwei Arten, als Private Equity Fund (»Heuschrecke») oder als "Systemspieler « auf Finanzmärkten (»trend followers«). In beiden Fällen werden kaum reale Werte geschaffen, sondern primär (Bewertungs-) Gewinne durch Umverteilungen erzielt. Gelingt es etwa einem Private Equity Fund, den Unternehmenswert durch Lohnkürzungen zu steigern, so steht dem höheren Barwert der künftigen Gewinne der geringere Barwert der künftigen Lohnzahlungen gegenüber.

Der Staat fördert diese Finanzakkumulation: Bewertungs- und Spekulationsgewinne sind steuerlich besser gestellt als Gewinne aus realwirtschaftlicher Tätigkeit, überdies profitieren Finanzsektor und Aktienmarkt von der Förderung der privaten Altersvorsorge. Die Politik der Europäischen Zentralbank (EZB) passt ins Bild: Den Interessen des Finanzkapitals wird der Vorrang gegenüber jenen der Realvermögen gegeben. Dies kommt im Primat des Geldwerts ebenso zum Ausdruck wie in den relativ zur Wachstumsrate hohen Realzinsen (etwa im Vergleich zu den USA oder Japan).

In diesem Essay (in doppeltem Sinne) möchte ich skizzieren, wie das Verhalten von Unternehmen, Haushalten und Staat unter finanzkapitalistischen Rahmenbedingungen zum Anwachsen der beiden Hauptprobleme Arbeitslosigkeit und Staatsverschuldung beigetragen hat. Dabei werde ich verschiedene Module meines Forschungsprogramms der letzten 25 Jahre in extrem kompakter Form zu einem Gesamtbild zusammenfügen. Dieses skizziert verschiedene »Querverbindungen « lediglich in groben Strichen. Die Argumentation kann daher schon aus diesem Grund nicht "zwingend « sein, aber (hoffentlich) anregend.

\section{Die Geschäfte mit Finanzderivaten}

Der größte Anteil aller Finanztransaktionen entfällt auf den Handel mit Derivaten, insbesondere mit Futures und Optionen. Dies sind Wetten auf die künftige Entwicklung eines Preises/Kurses, sei es von Anleihen (Zinssätze), Aktien, Rohstoffen, Agrarprodukten oder Devisen (Wechselkurse). Dabei macht der »Wetteinsatz« nur einen Bruchteil des (Basis-) Werts aus. Beispiel: Jemand erwartet einen Kursanstieg deutscher Aktien und kauft deshalb einen DAX-Future. Sein Wert beträgt $25 €$ je Indexpunkt, bei einem Indexwert von 6.000 also I50.000 €. Hinterlegen muss er beim Kauf nur eine Margin von etwa sieben Prozent, also I0.500€. Steigt nun der DAX um zehn Prozent und damit der Wert des Future auf I65.00o €, so macht der »Spieler« einen Gewinn von I43 Prozent. Sinkt der DAX aber um zehn Prozent, so sind die I0.500 € verloren und der Spekulant muss noch $4.500 €$ nachzahlen. Die Hebelwirkung (»leverage effect «) von I4,3 resultiert daraus, dass der Basis(kontrakt)wert I4,3 Mal so hoch ist wie der Wetteinsatz.

Mit einer Option erwirbt jemand das Recht, das zugrunde liegende Asset innerhalb einer Frist zu einem bestimmten (Ausübungs-)Preis zu kaufen (Call) oder zu verkaufen 
(Put). Erwartet ein Trader einen Anstieg des Eurokurses, so wird er eine Call-Option kaufen. Trifft seine Prognose zu, so wird der Preis der Option viel stärker steigen als jener des Basiswerts (der Eurokurs). Die Bandbreite des Hebels ist bei Optionen größer als bei Futures, da sie von verschiedenen Faktoren abhängt (Differenz zwischen aktuellem Kurs und Ausübungspreis, Stärke des Kurstrends, Volatilität des Kurses, Restlaufzeit der Option).

Amateurspekulanten lassen sich davon faszinieren, dass Optionspreise manchmal an einem Tag um 30 Prozent oder sogar 50 Prozent steigen. Allerdings sind Amateure nicht in der Lage, das Risiko von Optionsgeschäften abzuschätzen. Erstens ist die auf der Wahrscheinlichkeitstheorie basierende Bestimmung des "fairen « Optionspreises mathematisch anspruchsvoll. Zweitens hält sich die Realität häufig nicht an die Wahrscheinlichkeitstheorie. Letzteres ließ 1998 den Hedge Fund »Long-term Capital Management« mit Verlusten von fünf Mrd. US-\$ »crashen « (zu diesem »Betriebsunfall« vgl. Edwards I999 und Lowenstein 2000). ${ }^{\mathrm{I}}$

Während die börsennotierten Derivate auch von Amateuren gehandelt werden, sind bestimmte Geschäfte den professionellen Tradern vorbehalten. Dies betrifft insbesondere Spot- und Termingeschäfte im Devisenhandel sowie Swaps.

Bei einem Spotgeschäft kauft ein Devisenhändler mit einem Einsatz von durchschnittlich fünf Millionen US-\$ jene Währung, deren Aufwertung er erwartet (etwa den Euro), und verkauft mit Gewinn, wenn seine Prognose zutrifft. Den gleichen Effekt erzielt er durch einen Terminkontrakt: Er verpflichtet sich, zum heute fixierten Terminkurs von I,25 US-\$ in - angenommen - einer Woche vier Millionen $€$ zu kaufen. Ist der Eurokurs bis dahin auf I,28 US-\$ gestiegen, so streicht der Trader die Differenz zwischen dem Wert des Kontrakts (fünf Millionen US-\$) und dem Wert der vier Millionen $€$ zum neuen Kurs (5.I20.000 US-\$) als Spekulationsgewinn ein (I20.000 US-\$).

Ein Swap kombiniert eine Spot- und ein Termingeschäft: Jemand tauscht mit einem Partner zum aktuellen (Kassa-)Kurs vier Millionen $€$ gegen fünf Millionen US-\$ und verpflichtet sich zu einem späteren Gegentausch zum heute fixierten (Termin-)Kurs. Ein Händler verleiht etwa für eine Woche Eurodevisen und borgt dafür Dollardevisen. Wertet der Euro auf, so hat er gewonnen, wertet der Euro hingegen ab, so hat er verloren.

Der Wettcharakter ist bei Swaps besonders augenfällig. Diese Geschäfte erstrecken sich (deshalb) nicht nur auf Wechselkurse, sondern auch auf Zinssätze, Aktienkurse oder Rohstoffpreise. Swapverluste trugen wesentlich zu »Betriebsunfällen« wie jenen der Barings Bank, LTCM oder der BAWAG bei. Swaps verdeutlichen eine weitere Eigenschaft des Derivathandels: Er stellt ein Umverteilungsspiel dar, die Summe aller Gewinne entspricht der Summe aller Verluste wie bei Lotterien, Pferdewetten oder im Casino (nur die "Spielorganisatoren" gewinnen immer).

Ursprünglich sind Terminkontrakte zur Absicherung gegen das Risiko von Preisschwankungen landwirtschaftlicher Produkte entstanden (Hedging). Mit der Aufgabe fester Wechselkurse (197I/73), den nachfolgenden Schwankungen von Dollarkurs und Erdöl-

I Im Vorstand von LTCM saßen übrigens die Ökonomen Myron S. Scholes und Robert C. Merton, welche 1997 den Nobelpreis für die Entwicklung der Optionspreisformel bekommen hatten. 
preis (vgl. Abbildung 4, S. 82, und Abbildung 5, S. 84) und mit dem Anstieg der Zinssätze Ende der I970er Jahre (also im Zug der Liberalisierung der Finanzmärkte) stieg die Bedeutung von Wechselkurs-, Rohstoff- und Zinsderivaten, aus Gründen des »hedging« ebenso wie von »outright speculation«. Die Einführung von immer mehr an Börsen gehandelten Futures und Optionen (insbesondere auch auf Aktien) und die Entwicklung immer komplexerer Handelstrategien, welche beide Instrumente kombinieren, gaben dem Derivathandel in den I980er Jahren einen enormen Auftrieb (auch in Wechselwirkung mit dem Aktienboom, vgl. Abbildung 8). Treibende Kraft ist die Spekulation geworden, Hedging hat im Vergleich dazu nur geringe Bedeutung. Dies ergibt sich allein schon aus dem Handelsvolumen mit Derivaten.

\section{Das Handelsvolumen auf den Finanzmärkten}

Laut der Bank für Internationalen Zahlungsausgleich (BIZ) betrug die Summe der Basiswerte von Futures und Optionen bezogen auf verzinsliche Wertpapiere, Aktien und Devisen (= Finanzderivate) 2005 weltweit 58.520 Milliarden US-\$, die Terminkontrakte zwischen professionellen Akteuren hatten einen Wert von 270.I00 Milliarden US-\$. Der Gesamtwert von 328.620 Milliarden US-\$ ist etwa zehn Mal so hoch wie das BIP aller Industrieländer.

Abbildung I: Relation des Handelsvolumens der Finanzderivate zu nominellem BIP

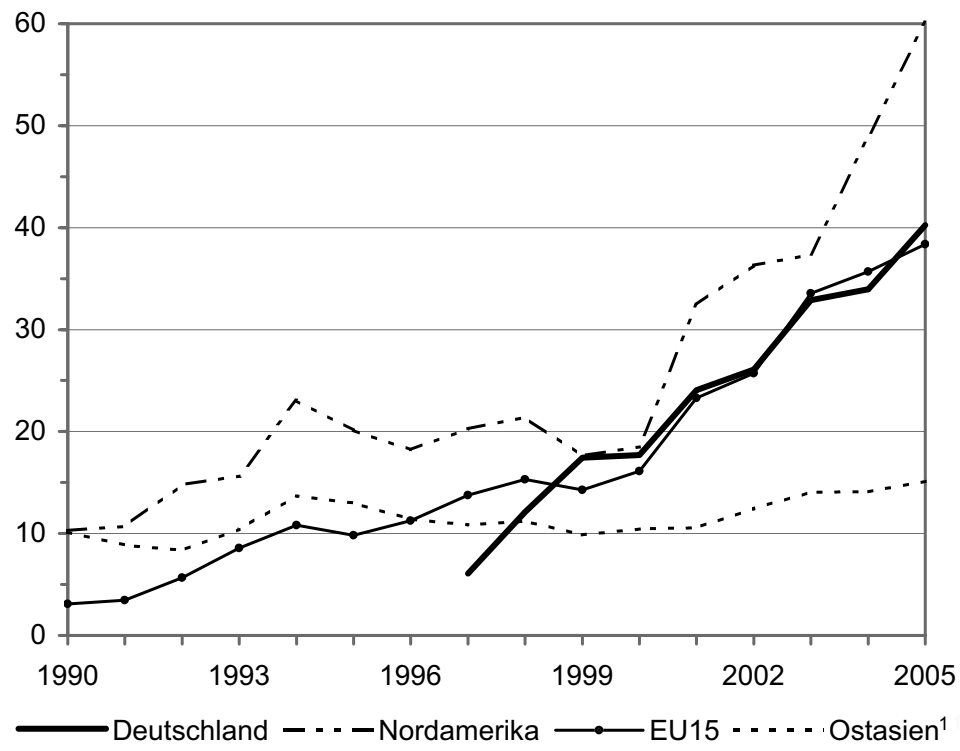

I Japan, Südkorea, Australien, Neuseeland

Quelle: BIS 
Futures und Optionen werden an den Börsen im Lauf ihrer "Lebenszeit« viele Male umgeschlagen. Dementsprechend ist ihr Handelsvolumen viel größer als ihre Basiswerte. Laut BIZ lag der "Turnover « von Finanzderivaten im Jahr 2005 weltweit bei I.408.379 Milliarden US-\$ (5.634 Milliarden US-\$ pro Handelstag). Berücksichtigt man noch die Tagesumsätze im Handel mit Devisen (I.900 Milliarden US-\$), Rohstoffderivaten (50 Milliarden US-\$), mit Anleihen (47 Milliarden US-\$) sowie Aktien (I68 Milliarden US-\$), so ergibt sich: Pro Tag wurden 2005 auf den Weltfinanzmärkten (Basis-)Werte in Höhe von etwa 7.80o Milliarden US-\$ umgesetzt. Dieser Wert ist $56 \mathrm{Mal}$ so hoch wie das BIP aller Industrieländer.

Das geringe Gewicht des Handels mit »echten « Aktien und Anleihen (Spottransaktionen) im Vergleich zu den Derivaten zeigt die enorme Bedeutung kurzfristig-spekulativer Geschäfte relativ zu längerfristiger Veranlagung. Darauf deutet auch der spektakuläre Zuwachs des Handels mit Finanzderivaten hin: Er stieg zwischen 1986 und 2005 weltweit um 20,5 Prozent pro Jahr.

Am größten ist das Handelsvolumen mit Finanzderivaten im Land mit der höchsten Spekulationskultur: In den USA ist es 6o Mal so hoch wie das BIP, I986 war es 6,5 Mal so hoch. Doch Europa holt auf: War der Turnover mit Finanzderivaten 1986 nur halb so hoch wie das BIP, war er 2005 etwa $38 \mathrm{Mal}$ höher (vgl. Abbildung I).

Innerhalb Europas wurde Deutschland zum wichtigsten Finanzplatz für diese Geschäfte: Seit ihrer Gründung im Jahr 1997 expandierten die Umsätze an der Derivatbörse EUREX um etwa 30 Prozent pro Jahr, 2005 waren sie bereits 40 Mal höher als das deutsche BIP.

\section{Ursachen der Expansion des Finanzhandels}

Welche Faktoren haben den Handel mit Finanzderivaten derart expandieren lassen?

Erstens: Zwischen der Instabilität der Kurse und dem Handelsvolumen besteht eine Wechselwirkung. Je stärker die (Derivat)Preise von Aktien, Rohstoffen, Währungen und Anleihen schwanken, desto größer sind (potentielle) Spekulationsgewinne, die wiederum Handelsvolumen und Kursinstabilität erhöhen. Letztere steigert auch den Bedarf nach Kursabsicherung und induziert so zusätzliche Derivattransaktionen.

Zweitens: Die Informationstechnologien haben die Transaktionsgeschwindigkeit drastisch gesteigert. Von allen Orten der Welt kann man via Internet auf den wichtigsten Börsen »in real time« handeln. Orders werden auf elektronischen Börsen wie der EUREX in Sekunden ausgeführt, die Transaktionskosten sind minimal. Gleichzeitig hat die Verwendung computergesteuerter Spekulationssysteme enorm zugenommen. Diese Spielsysteme verarbeiten auch sehr "schnelle» Daten (etwa im Minuten-Takt, vgl. Abbildung 3 ).

Drittens: Der Boom der Aktienkurse seit den frühen I980er Jahren hat gemeinsam mit den höheren Realzinsen die »Renditeansprüchlichkeit« erhöht. Unternehmen tätigen nur mehr solche Investitionen, welche den auf Finanzmärkten erzielbaren Renditen entsprechen. Hohe Unternehmensgewinne stimulieren daher nicht mehr die Realinvestition, sondern primär die Finanzakkumulation und -spekulation.

Viertens: Diese Entwicklung hat den Aktivitätsfokus der Banken verschoben. Investitionsfinanzierung oder das Kleinkundengeschäft verlieren an Bedeutung, zur wichtigs- 
ten Profitquelle werden »trading « und »asset management«. Dies gilt insbesondere für die beiden derzeit profitabelsten Bankhäuser, Deutsche Bank und Goldmann Sachs (vgl. The Economist 2006).

Fünftens: Viele Hedge Funds spezialisieren sich auf das computergestützte Ausnutzen kurzfristiger Kursschübe von Finanz- und Rohstoffderivaten. Steigt ein Kurs mit einer bestimmten Stärke, so produzieren die "trading systems« ein Kaufsignal, setzt eine Gegenbewegung ein, so wird zweimal verkauft (die »long position" wird geschlossen und eine "short position « eröffnet). Die Interaktion von tausenden solcher "Systemspieler « verstärkt die Kursschübe (vgl. Abbildungen 2 und 3, S. 82).

Sechstens: Die Zahl der Amateurspekulanten hat stark zugenommen. Für die USA kann man aus den Auflagen der Magazine über Derivatspekulation schließen, dass ihre Zahl die Millionengrenze übersteigt (»the dentists and doctors«). Nur wenige widmen sich als »day trader « ausschließlich der Spekulation. In Europa dürfte die Zahl der Amateurspekulanten nicht wesentlich kleiner sein als in den USA. Die enorme Vielfalt deutscher Websites und Newsletters mit Tipps für den Derivathandel deutet darauf hin, dass diese »Finanzspiele« in Deutschland besonders beliebt sind. Überdies bieten alle großen Banken ihren Privatkunden die Durchführung dieser Geschäfte an und veranstalten Seminare zur Spekulationsweiterbildung.

\section{Die wichtigsten Handelsstrategien}

Professionelle Trader basieren ihre Entscheidungen auf die Interpretation neuer Informationen (»news«), auf das "Ausreiten« von Trends (»trend followers«) oder auf den Wechsel in der Trendrichtung (»contrarian trading«). Im ersten Fall geht es darum, in Sekunden abzuschätzen, wie die anderen Marktteilnehmer auf eine Nachricht reagieren werden, die auf den Bildschirmen erscheint: Ist sie überraschend oder war sie schon »eingepreist«?

Beginnt der Kurs auf Grund »echter« News zu steigen, so generieren zuerst die verschiedenen »trend-following systems « auf der Basis von Hochfrequenzdaten (z.B. ZehnSekunden-Kurse) eine Sequenz von Kaufsignalen. Ihre Exekution treibt den Kurs weiter nach oben, es folgen die Kaufsignale der »langsameren« technischen Modelle usw.

Die Abbildungen 2 und 3 zeigen am Beispiel des US-Dollar/Euro-Wechselkurses, wie selbst ein simples technisches Modell Kurstrends ausnutzen kann, und zwar auf ganz unterschiedlichen Zeitskalen (Tages- bzw. Fünf-Minuten-Kursen): Wenn der aktuelle Kurs den gleitenden 35-Perioden-Durchschnitt von unten (oben) durchbricht, wird gekauft (verkauft).

Je länger ein Trend andauert, desto mehr verliert er an Dynamik (gegen Ende springen nur mehr Amateure auf). Auf dieser Erfahrung beruhen die »contrarian strategies«. Sie produzieren Verkaufssignale in der Spätphase eines Aufwärtstrends, spekulieren also auf sein "Kippen«. Die Exekution dieser Signale trägt dann zu einem Richtungswechsel bei. Für diese Handelsstrategien werden auch »Charttechniken « verwendet, also die Identifikation solcher Kurskonfigurationen, welche einen Trendwechsel signalisieren sollen (z.B. »head and shoulder $\lll$ ). 
Abbildung 2: Ein Spekulationsmodell auf Basis von Tageskursen des Wechselkurses US-Dollar je Euro (1999-2005); Tagesdaten

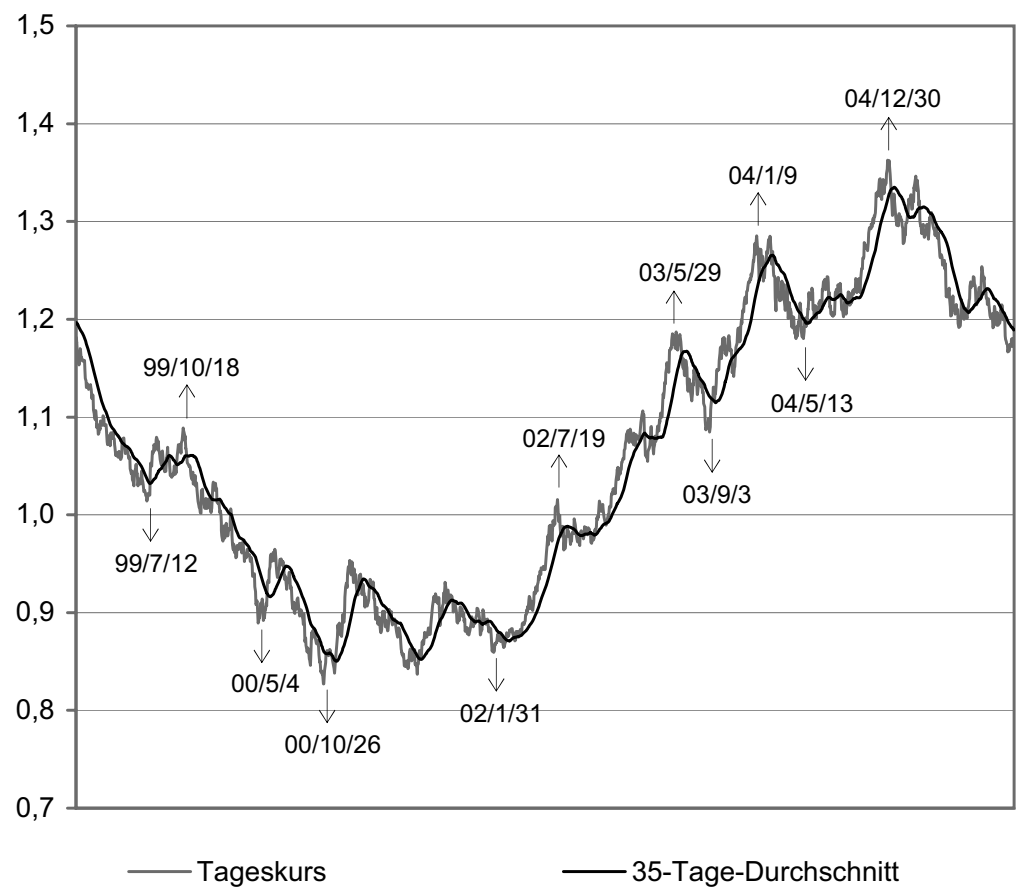

Quelle: Federal Reserve System, WIFO

Angesichts der Geschwindigkeit von Kursbewegungen und Transaktionen muss ein erfolgreicher Trader permanent auf Marktentwicklungen reagieren können (ein Devisenhändler hält eine offene Position in Höhe von üblicherweise fünf Millionen US-\$ im Durchschnitt nur zehn Minuten lang; vgl. Lyons I998). Bei Handelsschluss stellt er deshalb seine Positionen glatt. Lediglich höher gestellte »asset manager « dürfen strategische »overnight positions" halten, welche mittelfristige Trends auszunutzen versuchen.

Die meisten Handelssysteme produzieren häufiger Verluste als Gewinne. Allerdings ist der Durchschnittsgewinn der "winning trades« (auf Grund von Trends) viel höher als der Durchschnittsverlust der »losing trades« (auf Grund kleinere Kursschwankungen), sodass viele technische Modelle insgesamt profitabel sind (zu Verbreitung und Profitabilität technischer Spekulationssysteme vgl. Menkhoff/Taylor 2007 und Schulmeister 2006).

Schließlich erfordert das im Derivathandel besonders hohe Risiko ein entsprechendes Management. Offene (spekulative) Positionen müssen auf unterschiedliche Märkte und Derivattypen verteilt und phasenweise durch Gegenpositionen abgesichert werden (»hedging «). Die Minimierung des Gesamtrisikos relativ zum (potentiellen) Spekulationsgewinn stellt die größte Herausforderung für Finanzmathematiker und -manager dar. 
Abbildung 3: Ein Spekulationsmodell auf Basis von 5-Minuten-Kursen Wechselkurs USDollar je Euro (6.-I3. Juni 2003); 5-Minuten Daten (Olsen)

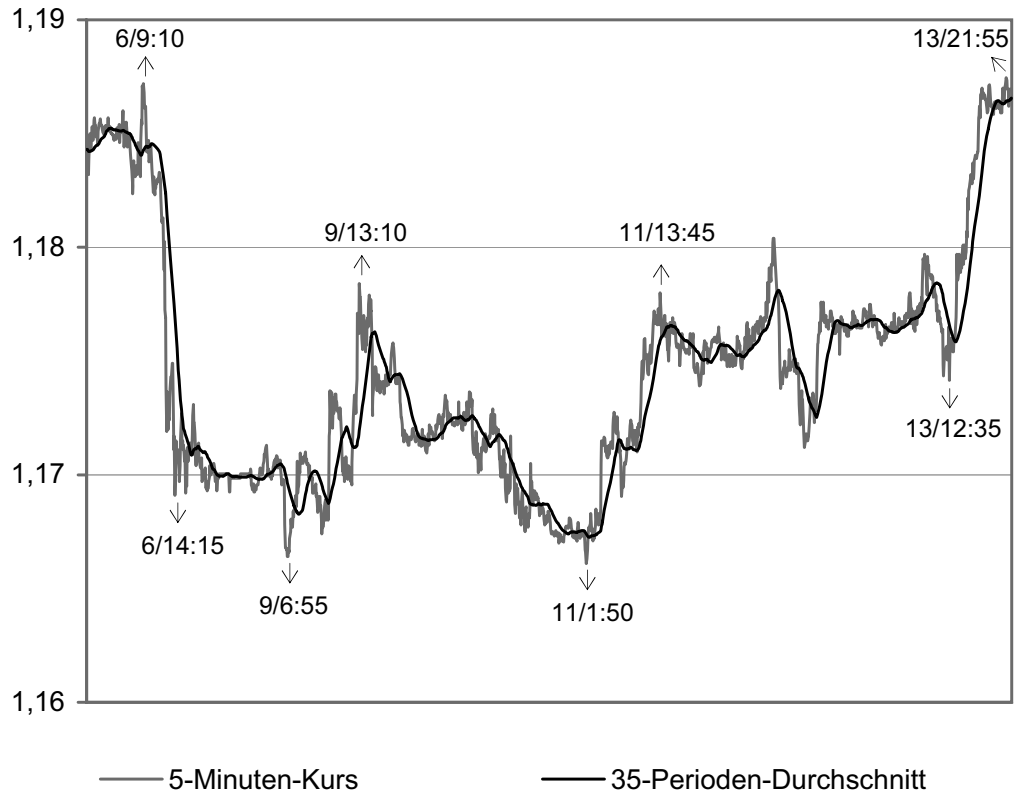

Quelle: Olsen Data, WIFO

\section{Die Gewinner und Verlierer im Finanzhandel}

Wer sind die Gewinner und Verlierer im Derivathandel? Die Banken und Hedge Fonds machen Gewinne, zwar nicht jede(r) einzelne, wohl aber in ihrer Gesamtheit. Die beiden profitabelsten Banken, Goldman Sachs und Deutsche Bank, dürfen für 2006 zumindest 25 bzw. 20 Milliarden US-\$ an Handelsgewinnen erwarten, der größte Teil aus Derivatgeschäften.

Da die professionellen Akteure in ihrer Gesamtheit gewinnen, müssen die Amateurspekulanten in ihrer Gesamtheit die Verlierer sein. Dafür sprechen vier Unzulänglichkeiten auf Seiten der Amateure: Sie können nicht permanent auf das Marktgeschehen reagieren, sie haben nicht genügend Kapital, um Verlustphasen durchzustehen, sie sind zu einem Risikomanagement weder finanziell noch intellektuell in der Lage, und schließlich haben sie viel schlechteren Zugang zu Insiderinformation als etwa Investmentbanken.

Ins Bild gesetzt: Relativ wenige »Hirten« (professionelle Trader) führen viele "Schafe» auf die Weide, dort werden sie geschoren, sei es von Banken oder Hedge Funds. Einzelne Schafe mögen tüchtig sein und andere scheren, als Herde aber müssen sie Federn lassen. So entsprechen den Gewinnen von vielleicht 20.000 "Profis" (Banken, Hedge Funds, Versicherungen, Industriekonzerne) die Verluste von Millionen Schafen, pro Schaf sind sie klein und können so verkraftet werden. Riesenschafe mit »Spieleinsätzen « von Profis, aber einem Spielverhalten von Amateuren kommen selten vor (wie Barings oder BAWAG). 
Die Umverteilung von den Amateuren zu den Profis des Finanzsektors kommt indirekt auch durch deren Einkommen zu Ausdruck: Bei Goldman Sachs beträgt die durchschnittliche Lohnsumme aller 24.000 Beschäftigten 520.000 US-\$ pro Jahr (vgl. The Economist 2006).

Wenn die Amateure in ihrer Gesamtheit die Verlierer sind, warum expandiert das Spiel weiter? Warum ziehen sich die geschorenen Schafe nicht zurück und dämpfen so das Geschäftsvolumen?

Erstens: Für viele vermittelt das Spiel an sich Spannung. Schließlich verlieren auch die meisten Lottospieler oder Casinobesucher ihren Einsatz und machen doch weiter.

Zweitens: Wenn auch ein Spekulationsverlust Leiden schafft, so fordert er gleichzeitig heraus, das nächste Mal den Markt zu besiegen (nur dieser erscheint als »Gegner», die mitspielenden Profis bleiben anonym).

Drittens: Die meisten Amateurspieler verfügen über genügend (Normal-)Einkommen, um das verlorene Spielkapital zu ersetzen.

Viertens: Den größten Effekt hat die vollständig asymmetrische Information über Gewinne und Verluste. Die Spekulationsmagazine sind voll von Artikeln »How I made a million « mit diesem oder jenem System, etwas diskreter legen auch die Trading-Seminare der Banken diese Möglichkeit nahe, und im Bekanntenkreis berichtet man stolz, wenn man "eingecasht" hat. Artikel über "How I lost a million« fehlen, und wer verspielt hat, behält es für sich.

Fünftens: Die Kunde von den Gewinnen und das Schweigen der Lämmer über ihre Verluste führen den Märkten stetig »frisches Blut« zu. Einzelne Verlierer lernen zwar das Richtige und ziehen sich zurück, der Zustrom an Eleven ist aber viel größer.

Fazit: Die individuellen und sozialen Verhaltensweisen unter Unsicherheit sind komplexer als Milton Friedmans eingängig-simples Argument, dass verlustbringende (und gleichzeitig destabilisierende) Spekulation keine Bedeutung haben könne, da sie durch erfolgreiche Spekulanten rasch aus dem Markt gedrängt würde (vgl. Friedman I953).

\section{Destabilisierung der wichtigsten Preise in der Weltwirtschaft}

Die Umverteilung durch den Derivathandel von der gehobenen Mittelklasse zu den Spitzenverdienern stellt kein gravierendes Problem dar (dies gilt auch für die interkontinentale Umverteilung zugunsten der USA, wo die besten Trader arbeiten). Zwei andere Effekte sind allerdings die wichtigsten langfristig wirksamen Ursachen für Arbeitslosigkeit und Staatsverschuldung in Europa:

- Die Spekulation auf den Derivatmärkten sowie im Devisenhandel destabilisiert die wichtigsten Preise in der Weltwirtschaft wie Wechselkurse, Rohstoffpreise (insbesondere den Ölpreis), Aktienkurse und Zinssätze.

- Die Unsicherheit über die Entwicklung dieser Preise und die hohen Finanzrenditen dämpft die realwirtschaftlichen Aktivitäten von Unternehmen, insbesondere Investitionen und (damit) die Schaffung neuer Arbeitsplätze. 
Auf welche Weise destabilisiert der Handel mit Derivaten ihre Preise und jene der zugrunde liegenden Assets? Unterschiedliche Spieler verwenden unterschiedliche Spekulationssysteme, angewendet auf unterschiedlichen Zeitskalen (von Zehn-Sekunden-Kursen bis zu Tageskursen). Die Konzentration von Kaufsignalen von »news-based traders« am Beginn eines Aufwärtstrends, gefolgt von Kauforders der "trend followers«, verstärkt und verlängert den Trend. Hat er an Dynamik verloren, so lassen ihn die Verkaufssignale der "contrarians« kippen (vgl. Schulmeister 2006).

Das Phänomen solcher Kursschübe lässt sich auf jeder Zeitskala beobachten. Aus ihrem Zusammenwirken ergeben sich mehrjährige Trends: Minutentrends in die gleiche Richtung, unterbrochen von kürzeren Gegenbewegungen, addieren sich zu einem Stundentrend, mehrere Stundentrends zu Tagestrends, usw. Insgesamt ergibt sich daraus die für alle "asset prices« typische Abfolge von Kursschüben, welche für einige Zeit in eine Richtung länger sind als die Gegenbewegungen, also ein stufenweiser Auf- oder Abwärtsprozess (vgl. Abbildungen 2 und 3). Auf Basis von Monatsdaten nimmt dieser Prozess die Gestalt mehrjähriger »bull markets« und »bear markets» an.

Abbildung 4: Wechselkurs US-Dollarje Euro und Kaufkraftparität (in Dollarje ECU bzw. Euro)

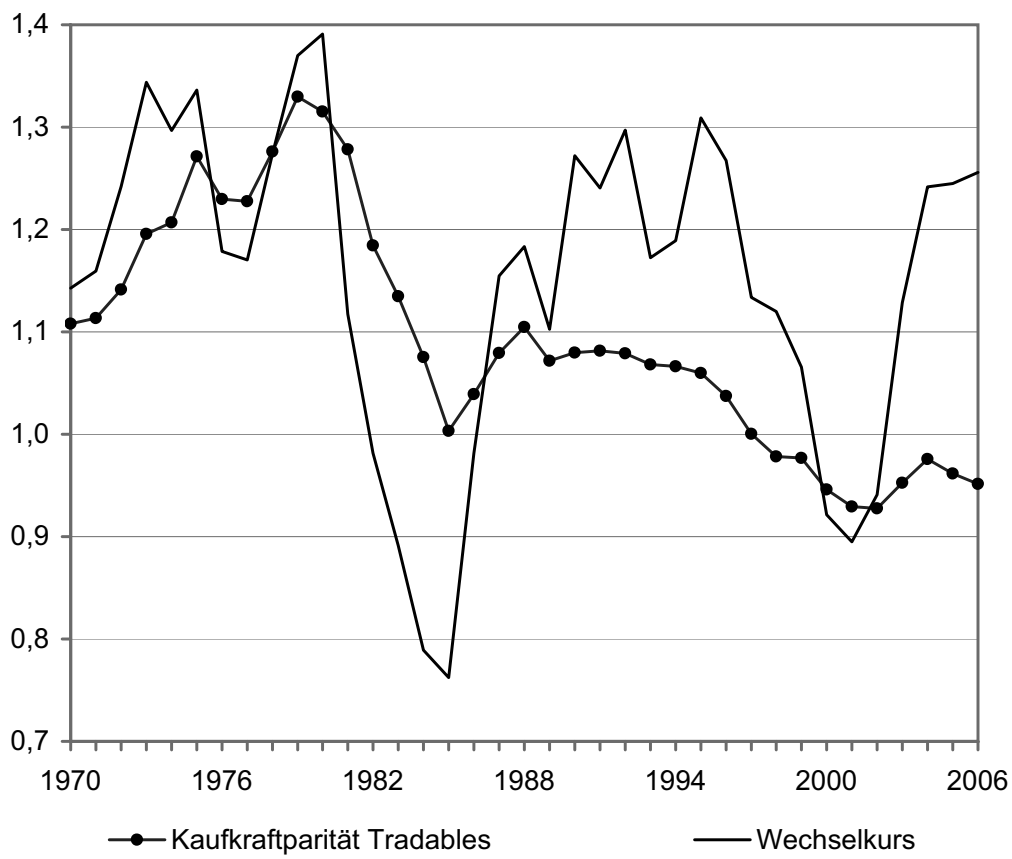

Quelle: OECD, Eurostat, Schulmeister 2005a

Aktuelle Beispiele für »bull markets« sind der Ölpreisanstieg sowie der Aktienboom seit 2003, wichtige "bear markets« waren der Aktienkursverfall 2000/03 sowie die Dollarabwertung 2002/04 (vgl. Abbildungen 4, 5, S. 84, und 8, S.87). 
Warum sind Kursschübe in eine Richtung für einige Zeit länger als die Gegenbewegungen? Hier kommen die Fundamentalfaktoren ins Spiel. Als etwa die US-Notenbank im Wirtschaftsabschwung 200I die Zinsen viel stärker senkte als die EZB und überdies US-Politiker ein »talking the dollar down « betrieben, während die EZB offenbar eine Stärkung des Euro begrüßte, signalisierte dies den Händlern: Der Dollar wird gegenüber dem Euro eher weiter abwerten als aufwerten, die Marktstimmung wurde/blieb »bearish«. Bei einem solchen »Erwartungsbias" wird mehr »Spielkapital « auf einen Abwertungsschub des Dollar gesetzt als auf einen Aufwertungsschub. Das gleiche gilt für strategische »overnight positions«.

Entscheidend für die destabilisierende Wirkung von Spekulation ist folgende Tatsache: Trader bilden auf Grund der "fundamentals « lediglich Erwartungen über die Bewegungsrichtung von Kursen, nicht aber über ihr Gleichgewichtsniveau. So wird ein Händler bei einer überraschenden Dollarzinssenkung blitzartig auf eine Euroaufwertung setzen (»Richtungserwartung ), statt das neue »Fundamentalgleichgewicht« des Euro-US-DollarKurses zu quantifizieren. Dies hätte nur dann einen Sinn, wenn jeder Trader damit rechnen könnte, dass alle anderen Trader zum gleichen Ergebnis kommen. In diesem Fall würde der Wechselkurs durch stabilisierende Spekulation zum neuen Gleichgewicht "springen" (dies nimmt die herrschende Theorie an). ${ }^{2}$

Technische Spekulationssysteme entsprechen der Erwartungsbildung in der Praxis des Finanzhandels, sie zielen lediglich darauf ab, hinlänglich oft die Richtung eines Trends zu erfassen. Die Komplexität der Entscheidungsfindung wird so auf jenes Minimum reduziert, das nötig ist, um Profite zu machen. Gleichzeitig trägt die Verwendung dieser Systeme entscheidend dazu bei, dass »asset prices« in mehrjährigen »bull markets u und »bear markets" ihre theoretischen Gleichgewichtsniveaus »überschießen«.

\section{Realwirtschaftliche Folgen instabiler "Asset Prices"}

Abbildung 4 zeigt die starken Abweichungen des US-Dollar/Euro(ECU)-Wechselkurses von seinem (theoretischen) Gleichgewichtswert der Kaufkraftparität international gehandelter Sachgüter und Dienstleistungen (zu seiner Berechnung vgl. Schulmeister 2005a). Zwischen 1980 und 1985 wertete der US-Dollar drastisch auf, die Unterbewertung des ECU wurde immer größer. Darauf folgte eine neuerlich überschießende US-Dollar-Abwertung, sodass der ECU in den I990er Jahren permanent überbewertet war. Dies wurde durch die Abwertung des ECU / Euro zwischen 1995 und 2000 korrigiert. Die mit der Rezession 200 I einsetzende Niedrigzinspolitik in den USA und ein begleitendes "talking the dollar down" trugen wesentlich zur neuerlich überschießenden Euro-Aufwertung bei (vgl. Abbildung 4).

2 Der Begriff "(de)stabilisierende Spekulation «, wie ihn Milton Friedman (1953) und die orthodoxe Theorie vertreten, macht keinen Sinn, wenn Akteure lediglich »Richtungserwartungen« bilden (statt "Niveauerwartungen«). In diesem Essay wird Spekulation dann/deshalb als »destabilisierend « bezeichnet, wenn /weil sie die Kurse in mehrjährigen, irregulären Zyklen um ihr (theoretisches) Fundamentalgleichgewicht als »Attraktoren« im Sinne der Chaostheorie schwanken lässt. 
Abbildung s: Dollarkurs und Erdölpreis

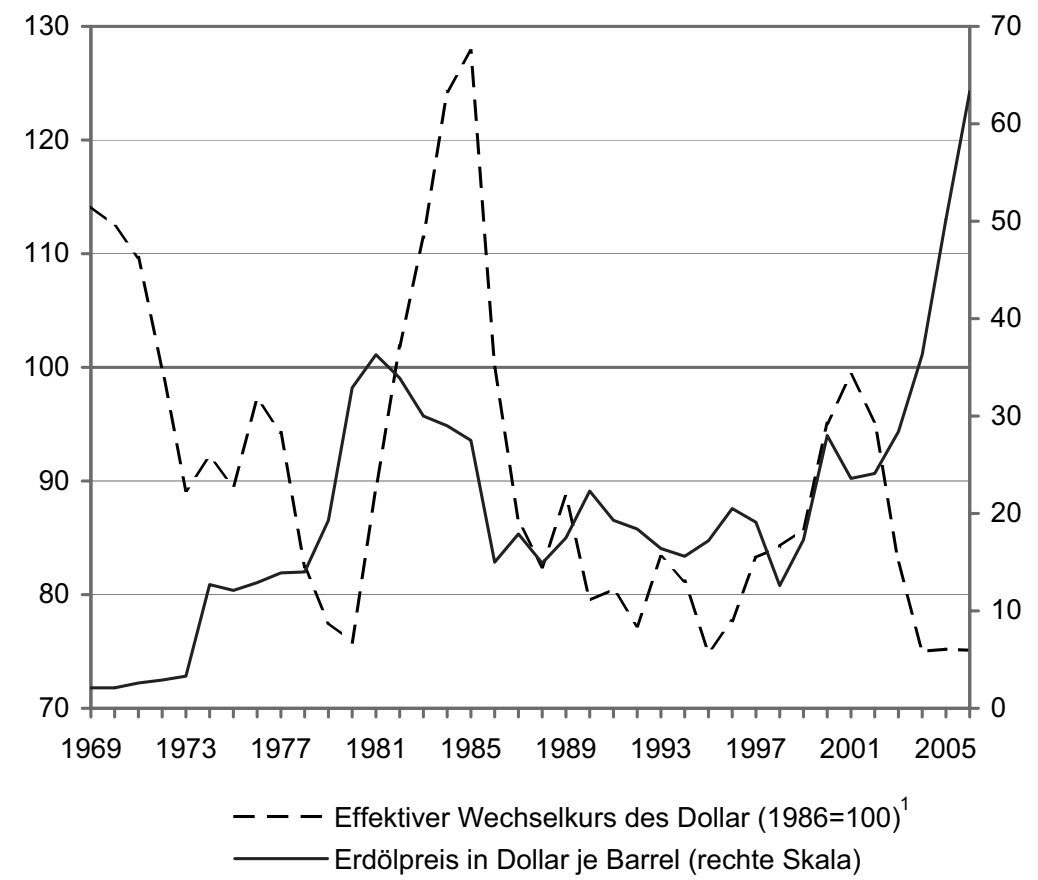

I Gegenüber DM, Franc, Pfund, Yen

Quelle: IMF, WIFO

Das mehrjährige Überschießen der Wechselkurse nach oben und unten ist nicht Folge einzelner "Schocks« und (zu) langsamer Annäherung an das Gleichgewicht (wie vom Mainstream angenommen), sondern Ausdruck der für Finanzmärkte typischen Dynamik: Die Kurse schwanken in irregulären Zyklen um die theoretischen Gleichgewichtswerte. Letztere wirken als "Attraktoren«, deren Anziehungskraft mit dem Ausmaß des "Overshooting" steigt. Bei bzw. nach (Wieder-)Annäherung an ihr (theoretisches) Gleichgewicht konvergieren spekulative Preise nicht zu diesem Niveau, sondern »durchschießen« dieses (vgl. Schulmeister 1987).

Die »long swings« der Wechselkurse haben drei Haupteffekte. Erstens, sie erhöhen generell Unsicherheit und Kosten von Handel und Direktinvestitionen. Zweitens, sie dämpfen die Exporte der Länder mit überbewerteter Währung. Davon ist der Euroraum besonders bei schwacher Konjunktur betroffen. Denn in diesen Phasen gelingt es der US-Politik, den US-Dollar niedrig zu halten (199I/94) oder sogar weiter "herunterzureden" (200I/04). Drittens, die besonders starken Schwankungen des Dollarkurses destabilisieren die Preise für Rohstoffe, insbesondere für Erdöl, da diese in US-Dollar notieren: Dollarabwertungen entwerten die realen Exporterlöse der Rohstoffproduzenten, diese versuchen in der Folge, höhere Dollarpreise ihrer Produkte durchzusetzen. Dies gelang dem OPEC-Kartell nach 
Abbildung 6: Dollarkurs und Welthandelspreise (I986=I00)

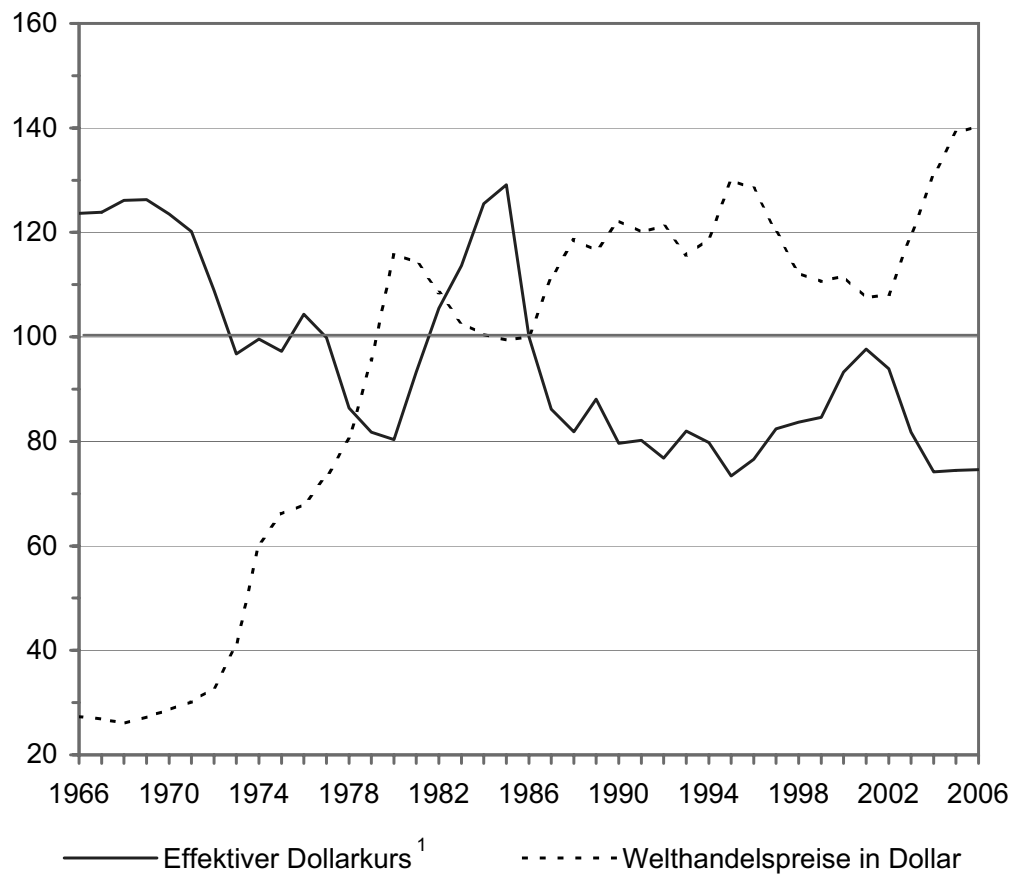

I Gegenüber DM, Franc, Pfund, Yen

Quelle: IMF, WIFO

den Dollarabwertungen 1971/73 sowie 1976/79 (vgl. Abbildung 6). Die beiden »Ölpreisschocks« lösten wiederum die beiden Rezessionen 1974/75 und 1980/82 aus. Auch in den vergangenen Jahren hat die starke Dollarabwertung zum Ausmaß der Erdölverteuerung beigetragen (vgl. Abbildung 5).

Die tendenziell gegenläufige Entwicklung von Dollarkurs und Rohstoffpreisen ist eine wichtige Ursache für den markant gegenläufigen Verlauf von Dollarkurs und Dollarpreisen im gesamten Welthandel (vgl. Abbildung 6). Dieses Phänomen ist wiederum die Hauptursache für die drastischen Schwankungen der Realzinsen für Auslandsschulden, da der größte Teil von ihnen in Dollar gehalten wird: In Phasen eines sinkenden Dollarkurses und hoher Welthandelsinflation ist der Realzins internationaler Dollarschulden (LIBOR minus Veränderungsrate der Welthandelspreise in US-\$) stark negativ wie in den I97oer Jahren - dies erleichterte es den Entwicklungsländern, ihre Auslandsverschuldung enorm auszuweiten (vgl. Abbildung 7). In Phasen eines steigenden Dollarkurses und sinkender Welthandelspreise ist der Realzins hingegen exorbitant hoch, wie I98I/85 (I4,7 Prozent) oder I996/200I (9,7 Prozent). Sein Anstieg nach Phasen einer »billigen« Welt(kredit)währung war Hauptursache der internationalen Schuldenkrisen 1982 sowie 1997 bis 1999. Die starke Entwertung der Weltwährung und die damit verbundene Inflationierung der Dollarpreise 
Abbildung 7: Realzins für internationale Dollarschulden (in Prozent)

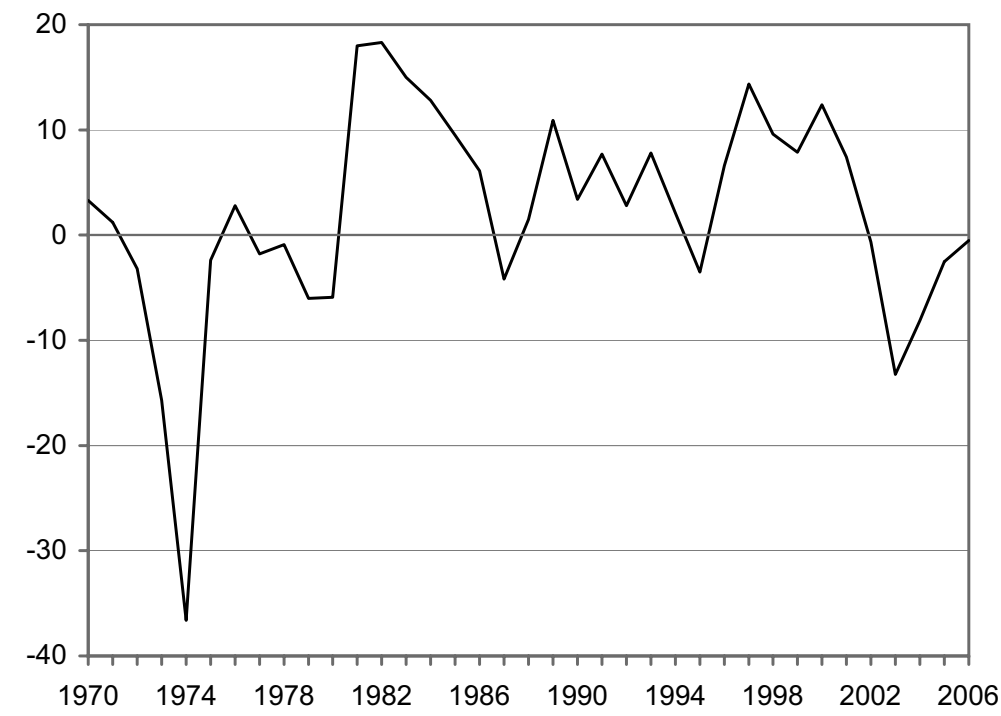

Quelle: IMF, WIFO

im Welthandel ließ den Realzins zwischen 2002 und 2005 wieder negativ werden (vgl. Abbildungen 6 und 7). Dies hat entscheidend zur Verbesserung der Finanzlage der verschuldeten Entwicklungsländer beigetragen. ${ }^{3}$

Die »manisch-depressiven « Schwankungen von "asset prices« sind bei Aktienkursen besonders markant ausgeprägt, und damit auch ihre Abweichungen von den realwirtschaftlichen »fundamentals» (vgl. Abbildung 8$): 4$

- In den I96oer und I970er Jahren stagnierten die Aktienkurse in Deutschland, gleichzeitig konzentrierte sich das Profitstreben der Unternehmen auf Realinvestitionen. Dementsprechend wuchsen der Realkapitalstock des "non-financial business" und sein Netto-Gesamtwert (= Realkapital zu laufenden Wiederbeschaffungspreisen minus Netto-Finanzverbindlichkeiten) viel stärker als Aktienkurse und Marktkapitalisierung (vgl. Abbildung 8).

- In der Periode zwischen 1982 und 1999 ergab sich eine spiegelverkehrte Entwicklung: Der Aktienboom ließ Kurse und Börsenwerte der Aktiengesellschaften viel rascher

3 Die Ursachen der Schwankungen des Wechselkurses des Dollar und ihr Ausmaß ergeben sich aus der Doppelrolle des Dollar als nationale Währung der USA (geprägt von »nationalökonomischen« Interessen der USA) und als Weltwährung (geprägt von der Funktion des Dollar als »numeraire« internationaler Flows und Stocks). Diese Zusammenhänge sowie ihre Konsequenzen für Rohstoffpreise, Welthandelsinflation und Finanzkrisen werden untersucht in Schulmeister (2000).

4 Zum Zusammenhang zwischen Realakkumulation, Finanzakkumulation, Aktienkursen und Börsenwerten der nicht-finanzielle Unternehmen in den USA und Deutschland seit I960 vgl. Schulmeister (2003). 
anwachsen als ihr Realkapitalstock bzw. ihr Netto-Gesamtwert. Diese Entwicklung wurde durch den nachfolgenden Verfall der Aktienkurse teilweise korrigiert, seit 2003 hat wieder ein markantes "Overshooting « der Aktienkurse eingesetzt. ${ }^{5}$
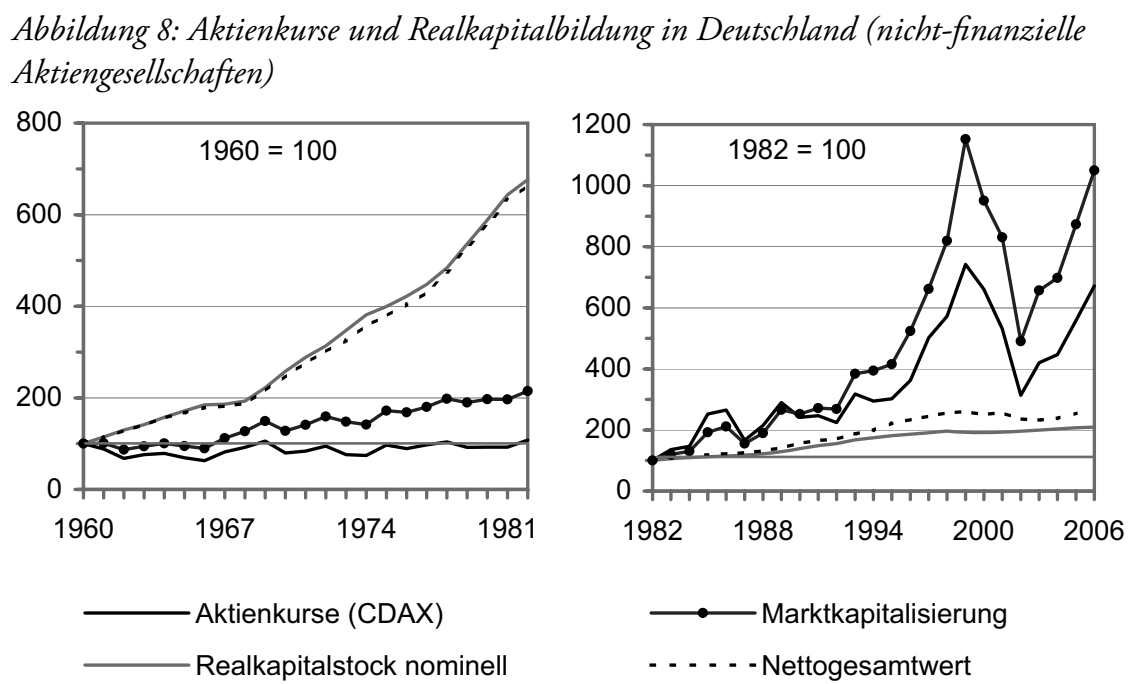

Quelle: Federal Reserve Bank, Deutsche Bundesbank, Statistisches Bundesamt Wiesbaden

\section{Unternehmer als "Finanzkapitalisten" und die Arbeitslosigkeit}

Die Instabilität von Aktienkursen, Rohstoffpreisen, Wechselkursen und Zinssätzen erhöhten die Unsicherheit hinsichtlich der Rendite längerfristig orientierter Projekte in der Realwirtschaft, daher beanspruchen die Unternehmen dafür eine höhere Risikoprämie. Gleichzeitig steigerte die Instabilität der Finanzmärkte die (potentiellen) Renditen von Finanzinvestitionen. Folge: Es werden nur mehr solche Realinvestitionen getätigt, welche den aus beiden Gründen höheren Renditeanforderungen genügen. Das Profitstreben des »nonfinancial business « hat sich somit in den vergangenen 25 Jahren von der Realkapitalbildung zu Finanzveranlagung und -spekulation verlagert. Dieser »schleichende« Prozess stellt die »strukturelle« Hauptursache für das langsame Anwachsen der Arbeitslosigkeit dar.

In der herrschenden Wirtschaftstheorie wird angenommen, dass das Beschäftigungsniveau von Niveau und Streuung der Reallöhne, vom Grad der Deregulierung der Arbeitsmärkte und von den sozialstaatlichen »Anreizen zu Arbeitslosigkeit» (Arbeitslosenunter-

5 Vermögenseffekte durch den Anstieg von Aktienkursen und (noch mehr) von Immobilienpreisen stimulieren den Privaten Konsum in den angelsächsischen Ländern wesentlich stärker als etwa in Deutschland (vgl. Schulmeister 2004). Der Preis für die Konsumbelebung via Aktienboom besteht in "Kurskorrekturen" wie 2000/2003 und ihren realwirtschaftlichen Folgen. Deutschland bezahlte diesen Preis, ohne davor die Vermögenseffekte lukriert zu haben. 
stützung, Sozialhilfe, etc.) abhängt, nicht aber von der Realkapitalbildung. Dies ist ein verhängnisvoller Irrtum. Neue Arbeitsplätze, welche dem europäischen Produktivitäts- und Einkommensniveau entsprechen, brauchen eine erhebliche Kapitalausstattung. Die Verlagerung der unternehmerischen Gewinnstreben von der Real- zur Finanzkapitalbildung ließ zuwenig produktive, mit entsprechendem Kapital ausgestattete Arbeitsplätze entstehen. Unter diesen Bedingungen musste die Arbeitslosigkeit steigen, sie ist ja nichts anderes als ein Defizit an Arbeitsplätzen.

Dass die Schaffung von Arbeitsplätzen eine dynamische Realkapitalbildung voraussetzt, zeigt ein empirischer Befund. Untersucht man das Einsatzverhältnis von Kapital und Arbeit in zehn Sektoren sowie in der Gesamtwirtschaft in den USA, Deutschland und Japan seit I960, so ergibt sich: In jedem einzelnen Jahr, in jedem Sektor und in jedem der drei Volkswirtschaften ist die Kapitalintensität gestiegen (vgl. Schulmeister 1998). ${ }^{6}$ Die Kapitalausstattung je Arbeitsplatz wird somit stetig erhöht und steigert so die Produktivität der Arbeitskräfte. Diese Entwicklung wird vom (prozess-)technischen Fortschritt bestimmt, nicht aber von den Faktorpreisen. So werden etwa PKW oder andere Industriewaren in China, Japan und Deutschland annähernd gleich kapitalintensiv produziert. Gleichzeitig sind der prozesstechnische Fortschritt und damit der monotone Anstieg der Kapitalausstattung je Arbeitsplatz irreversibel (keinerlei Lohnsenkung kann eine Rückkehr von Schweißrobotern zu Handschweißgeräten induzieren).

Aus diesen Gründen zeigt sich empirisch keinerlei Zusammenhang zwischen den relativen Kosten von Kapital und Arbeit und dem relativen Faktoreinsatz (vgl. Abbildung 9für die einzelnen Sektoren in den drei untersuchten Länder zeigt sich ein qualitativ nahezu gleiches Bild). Starke Verbilligungen des Faktors Arbeit wie Ende der 1970er oder 1980er Jahre als Folge einer Hochzinspolitik) hatten nicht einmal einen leicht dämpfenden Effekt auf den stetigen Anstieg der Kapitalintensität.

Eine mit der empirischen Evidenz konsistente Produktionsfunktion ist »dynamisiertlinear-limitational « (vgl. Schulmeister 1998): Kurzfristig erfolgen Outputvariationen entlang eines Prozessstrahls (zusätzliche Arbeitsplätze setzen eine proportionale Kapitalerhöhung bei konstanter Technologie voraus), längerfristig steigt die Kapitalintensität in Abhängigkeit vom technischen Fortschritt (der Anstieg des Prozessstrahls in der K,L-Ebene nimmt monoton zu). Das Niveau der Kapitalintensität variiert nach Sektoren, Branchen und Unternehmen; am höchsten ist die Kapitalausstattung je Arbeitsplatz in den Bereichen Energie, Verkehr und Nachrichten, Banken und Versicherungen und in der Industrie, am niedrigsten im Handel, Gastgewerbe und bei den persönlichen Diensten.

Bei konstanter sektoraler Produktionsstruktur kann Arbeitslosigkeit nur dann verringert werden, wenn der Kapitalstock rascher wächst als das Arbeitsangebot, und zwar zumindest im Ausmaß des Wachstums der Kapitalintensität. Dieses Differential muss ent-

6 Ein Update des für diese Studie entwickelten Datenbestands konnte für diesen Essay nicht durchgeführt werden. Für den gesamten Zeitraum 1960 bis 1995 erwiesen sich die wesentlichen Zusammenhänge als sehr stabil. Es ist daher unwahrscheinlich, dass sich in den vergangenen zehn Jahren eine andere Entwicklung ergab. 
Abbildung 9: Kosten und Einsatz von Kapital und Arbeit in Deutschland (1980=100)

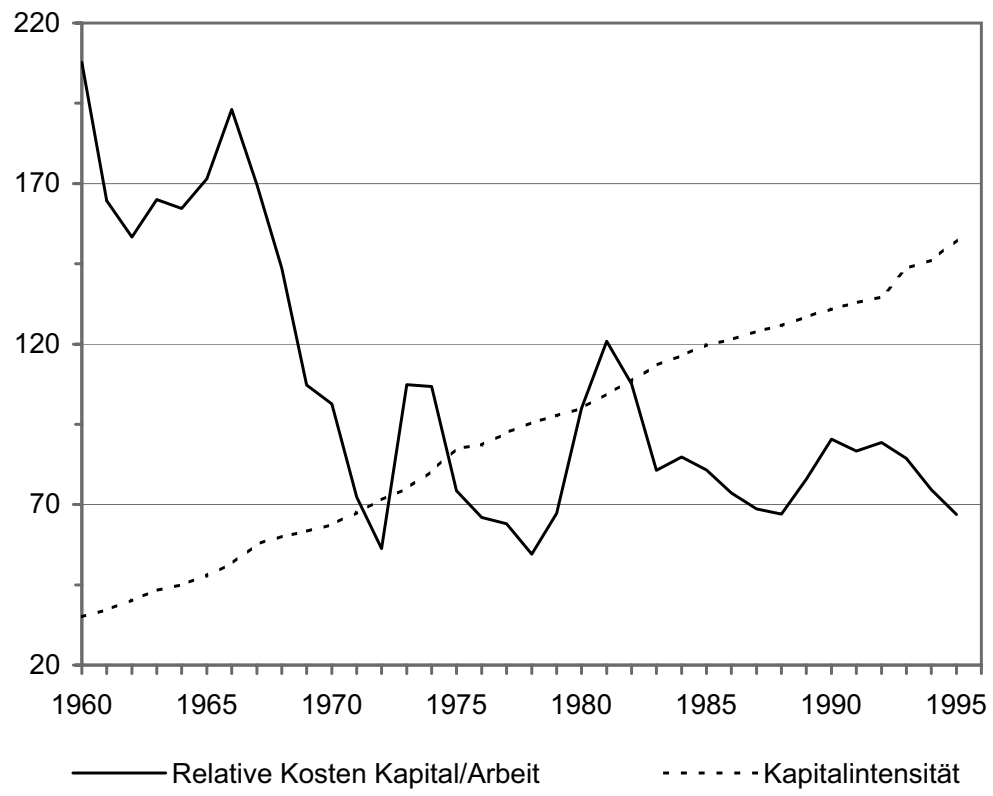

Quelle: OECD, IAB, DESTATIS, Schulmeister 1998

sprechend größer sein, wenn gleichzeitig ein Strukturwandel zu den Sektoren mit höherer Arbeitsproduktivität und höherer Kapitalintensität stattfinden soll (nicht zuletzt aus Gründen der technologischen Wettbewerbsfähigkeit).

Umgekehrt erfordert die Schaffung von Arbeitsplätzen in Sektoren mit geringerer Kapitalausstattung je Arbeitsplatz nur eine relativ schwächere Realkapitalbildung. In diesem Fall sind Arbeitsproduktivität und Realeinkommen entsprechend niedriger. Reallohnsenkungen sind allerdings keine hinreichende Bedingung für die Schaffung bzw. Ausweitung von Niedriglohnsektoren. Diese setzten vielmehr eine entsprechende Nachfragestruktur (und damit Einkommensverteilung) sowie ein entsprechend hohes Angebot an schlecht qualifizierten Arbeitskräften voraus (beide Bedingungen sind in der Wirtschaft der USA in höherem Maß gegeben als in Deutschland; vgl. Schulmeister 1998).

In der ersten Hälfte der Nachkriegszeit konzentrierte sich das unternehmerische Gewinnstreben auf Realinvestitionen und damit indirekt auf die Schaffung von Arbeitsplätzen. Diese wurden mit immer mehr Kapital ausgestattet und ermöglichten so einen steten Anstieg von Arbeitsproduktivität und Reallöhnen. Voraussetzung dafür waren "realkapitalistische" Rahmenbedingungen: Ein enges Bündnis zwischen Unternehmern und Gewerkschaften, das »Ruhigstellen« des Finanzkapitals (feste Wechselkurse, niedrige Zinssätze, regulierte Finanzmärkte), ein wirtschafts- und sozialpolitisch aktiver Staat, Dominanz der keynesianischen Wirtschaftstheorie und »Soziale Marktwirtschaft « als gesellschaftspolitisches Leitbild (auch als Reaktion auf die Bedrohung durch den »realen Sozialismus«). 
Mit dem Übergang vom Realkapitalismus zum Finanzkapitalismus im Verlauf der I970er Jahre musste sich die Dynamik von Realakkumulation, Wirtschaftswachstum und Beschäftigung nachhaltig abschwächen. ${ }^{7}$ Denn finanzkapitalistische Rahmenbedingungen verlagern das Gewinnstreben zu Finanzveranlagung und -spekulation, insbesondere über folgende Kanäle: Liberalisierung der Finanzmärkte, Destabilisierung der wichtigsten, zwischen der Real- und Finanzwirtschaft vermittelnden (Wechselkurse, Aktienkurse, Zinssätze), Zunahme von "Renditeansprüchlichkeit« und »short terminism«, Rückzug des Staates von einer aktiven Wirtschafts- und Sozialpolitik und Hegemonie der neoliberalen Wirtschaftstheorie.

Die USA wurden durch den »Regimewechsel« vom Real- zum Finanzkapitalismus weniger beeinträchtigt als Europa:

- Finanzkapitalistische Rahmenbedingungen entsprechen der »Wirtschaftskultur « in den USA in höherem Maß als in Europa - abgesehen von Keynes' „City“ (of London) existierte in Europa bis vor etwa 25 Jahren keine der "Wall-Street-Kultur« vergleichbare, relativ eigenständige »Finanzwelt « (kontinentaleuropäische Banken waren traditionell eng mit der Großindustrie verbunden, teilweise auch als deren Eigentümer).

- Die USA passen sich dem Mangel an hochproduktiven Arbeitsplätzen durch Schaffung von Niedriglohnarbeitsplätze an, die mit wenig oder keinem Realkapital ausgestattet sind ("working poor«-Arbeitsplätze). Diese Anpassung wurde durch den hohen Anteil relativ schlecht ausgebildeter Schulabgänger, das Ausmaß der Ungleichheit in der Einkommensverteilung (sie fördert die Nachfrage nach persönlichen Dienstleistungen) und den hohen Stellenwert von »Jeder ist seines Glückes Schmied « erleichtert (vgl. Schulmeister 1998).

- Zusätzlich vollzogen die USA um I990 einen Kurswechsel zu einer antizyklischen und wachstumsorientierten Geld- und Fiskalpolitik, auch die öffentliche Beschäftigung wird stetig ausgeweitet, insbesondere bei schwacher Konjunktur (von den zwischen 2000 und 2005 geschaffenen I,5 Millionen Arbeitsplätzen entfiel eine Million auf den öffentlichen Dienst).

- Die US-Politik lenkt das spekulative Gewinnstreben auf die Aktienkurse. Die für die Realwirtschaft wichtigeren Zinssätze und Wechselkurse werden auf niedrigem Niveau gehalten, insbesondere in Phasen schwacher Konjunktur (vgl. Schulmeister 2005b).

In Europa wurde das Land mit der traditionell fortgeschrittensten »realkapitalistischen Wirtschaftskultur «, also Deutschland, vom Finanzkapitalismus am stärksten beeinträchtigt (ähnlich wie Japan Ende der I98oer Jahre). Die nicht-finanziellen Unternehmen investierten während des Aktienbooms der I99oer Jahre in wachsendem Ausmaß in Finanzvermögen statt in Realkapital, nicht zuletzt auch durch Rückkauf der eigenen Aktien (vgl. Abbildung Io). Die Diskrepanz zwischen »echtem« Unternehmenswert und der Börsenbewertung weitete

7 Zur Unterscheidung zwischen diesen beiden Ausprägungen einer Marktwirtschaft und zu den Ursachen für den Wechsel von realkapitalistischen zu finanzkapitalistischen Rahmenbedingungen in den I970er Jahren vgl. Schulmeister 2004. 
sich (noch) stärker aus als in den USA. Dementsprechend stürzte der DAX zwischen 200I und 2003 viel stärker ab als die US-Aktien, nämlich um 70 Prozent (vgl. Abbildung 8). Dies entwertete das von den Unternehmen akkumulierte Finanzkapital, die massive Steuersenkung 200 I wurde daher nicht für Realinvestitionen, sondern zur finanziellen Konsolidierung verwendet.

\section{Abbildung Io: Real-und Finanzvermögen der nicht-finanziellen Kapitalgesellschaften} in Deutschland (in Prozent der Nettowertschöpfung)

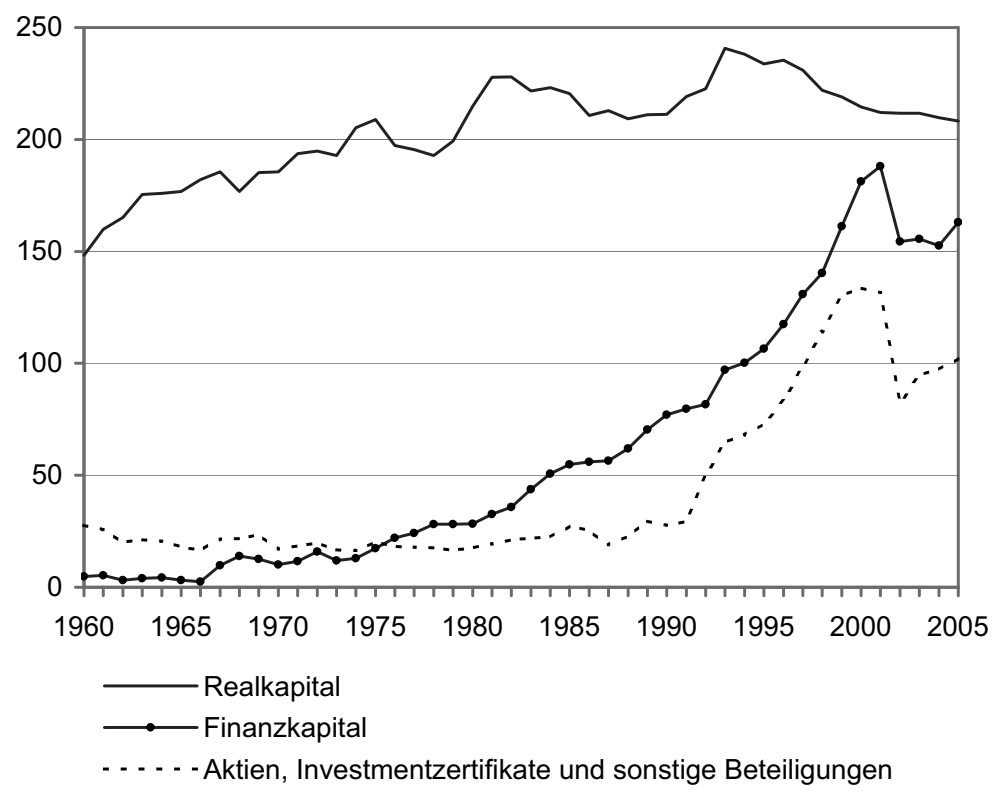

Quelle: Federal Reserve Bank, Deutsche Bundesbank, Statistisches Bundesamt Wiesbaden

Der Staat senkte die öffentliche Beschäftigung Is Jahre lang, 2005 war ihr Anteil an der Gesamtbeschäftigung um ein Viertel niedriger als in den USA (I2,I Prozent gegenüber I6,2 Prozent). Die Aufwertung des Euro gegenüber dem Dollar um mehr als 50 Prozent seit 2002, die Verteuerung von Erdöl und die "Verlockungen« der seit 2003 wieder boomenden Aktienkurse (der DAX ist seither um etwa 200 Prozent gestiegen; vgl. Abbildung 8, haben die Investitionsbereitschaft der deutschen Unternehmen weiter gedämpft und ihre Finanzveranlagungen und -spekulationen stimuliert. In einer Rückkoppelung steigert die kurzfristige (Derivat)Spekulation das Ausmaß der Euro-, Erdöl- und DAX»bull markets».

Die Eliten in Deutschland betrachten die Welt (derzeit) mit »neoliberaler Brille«. Das hat gravierende Folgen. Erstens, die Eliten können die steigende Arbeitslosigkeit nicht als Mangel an Arbeitsplätzen infolge unzureichender Realkapitalbildung wahrnehmen. Zweitens, sie können die stagnierende Realkapitalbildung bei (sehr) guter Gewinnentwicklung 
nicht als Folge steigender Gewinnchancen auf den (derivativen) Finanzmärkten erkennen. Drittens, sie können die Aktivitäten auf diesen Märkten nicht als die wichtigsten Preise destabilisierende »Umverteilungsspiele« wahrnehmen. Viertens, die Eliten können die "Abstinenz« des Staates von einer aktiven Wirtschaftspolitik nicht als zusätzliche Krisenursache erkennen.

Also hat die Politik in Deutschland auf den Anstieg der Arbeitslosigkeit mit der Reduktion der Arbeitslosenunterstützung (Hartz IV) und der massiven Förderung prekärer Arbeitsverhältnisse reagiert (Ich-AGs, Ein-Euro-Jobs, etc.). Die Arbeitslosen wurden dadurch billiger, die Arbeitenden auch, die Zahl sozialversicherter Jobs sank, jene der atypischen Jobs stieg. Mit all diesen Maßnahmen passte sich das System an den Mangel an (produktiven) Arbeitsplätzen als Folge unzureichender Realkapitalbildung an.

Produktionstheoretisch betrachtet: Die Arbeitslosigkeit soll durch Schaffung von »working poor-Arbeitsplätzen « verringert werden, welche mit wenig Kapital ausgestattet und daher auch wenig produktiv sind (der US-amerikanische Weg, insbesondere in den r98oer Jahren). Für einen nachhaltigen Erfolg dieses Rezepts sind allerdings Sozialstaat und Gewerkschaften in Deutschland (noch) zu stark, das Bildungssystem (noch) zu gut, die Einkommensverteilung (noch) nicht ungleich genug und die Wirtschaft generell (noch) nicht "finanzkapitalistisch" genug.

\section{Finanzkapitalismus und Staatsverschuldung}

Die Verlagerung des unternehmerischen Gewinnsstrebens von real- zu finanzwirtschaftlichen Aktivitäten seit Anfang der I980er Jahre wurde auch durch den Wechsel im Verhältnis von Zinssatz zu Wachstumsrate gefördert. Bis I980 setzten die Notenbanken die Leitzinsen in einer solchen Weise, dass die Kredit- und Anleihenzinsen niedriger waren als die Wachstumsrate. Auf Grund des »dynamic budget constraint« ergibt sich, dass bei dieser Konstellation Schuldner wie Unternehmen oder Staat permanent mehr (Investitions-)Kredite aufnehmen können als sie an Zinsendienst für die schon bestehenden Schulden zu leisten haben, ohne dass ihre Schulden rascher expandieren als das Bruttoinlandsprodukt (Schuldner können ein Primärdefizit aufrecht erhalten). Diese Bedingung fördert daher die Realkapitalbildung.

Um I980 haben die Notenbanken die Leitzinsen derart drastisch erhöht, dass das Zinsniveau die Wachstumsrate deutlich überstieg. Dies dämpfte die Investitionen der Unternehmen, weil ihre Neuverschuldung nunmehr niedriger gehalten werden musste als der Zinsendienst für die "Altschulden«. Statt zu investieren kauften die Unternehmen selbst Finanzaktiva, darunter auch Anleihen des Staates. Dessen Schulden mussten unter diesen Bedingungen stärker steigen als bei einem negativen Zins-Wachstums-Differential.

In den USA führte der geldpolitische Kurswechsel (Abkehr vom Monetarismus) Ende der I980er Jahre dazu, dass der Leitzins seither in "Normaljahren" der Wachstumsrate entspricht, in und nach Rezessionen wird er für mehrere Jahre deutlich tiefer gesetzt. In Deutschland liegt hingegen das Niveau der Leitzinsen (und noch mehr der Kredit- und Anleihenzinsen) seit 26 Jahren permanent über der Wachstumsrate. 
Folgende Kausalkette entwickelt sich unter finanzkapitalistischen Rahmenbedingungen (siehe dazu auch Abbildung I2, S. 96): ${ }^{8}$

- Aktienboom, kurzfristige (Derivat-)Spekulation und das die Wachstumsrate übersteigende Zinsniveau dämpfen Realinvestitionen und Wirtschaftswachstum.

- Steigende Arbeitslosigkeit und gedämpfte Steuereinnahmen lassen Budgetdefizit und Staatsverschuldung anwachsen.

- Der Staat verfolgt darauf eine Sparpolitik: Gekürzt werden die Ausgaben für öffentliche Investitionen und Beschäftigung sowie für die Kernbereiche des Sozialstaats, Gesundheitswesen, Pensionssystem, Bildungswesen und Arbeitslosenversicherung.

- Die Haushalte reagieren auf die damit verbundene Verunsicherung mit einer Stagnation der Konsumnachfrage, welche das Wirtschaftswachstum zusätzlich dämpft.

- Im Bereich der Altersvorsorge wird eine weitere Rückkoppelung wirksam: Die Unsicherheit über die sozialstaatliche Pension und die öffentliche Förderung privater Vorsorge dämpft nicht nur den Konsum, sondern stimuliert zugleich den Aktienboom.

Diese Zusammenhänge lassen sich auch als Interaktion der Investitions- und Sparpläne der wichtigsten Sektoren einer Volkswirtschaft darstellen. Die Unternehmen senken ihre Investitionen, sie nehmen daher weniger Kredite auf und akkumulieren Finanzvermögen: ihr Finanzierungsdefizit sinkt. Die Haushalte erhöhen ihr Sparen, ihre Überschüsse steigen. Der Staat versucht zu sparen, »erleidet« aber wachsende Defizite. Insgesamt übersteigen die Sparpläne die Investitionspläne, die Volkswirtschaft versucht also, "unter ihren Verhältnissen« zu leben, und schrumpft. Wenn allerdings das Ausland gleichzeitig bereit ist, seine Netto-Ausgaben für Produkte unseres "Musterlandes« in dem Maß zu steigern, das seinem überschüssigen Sparen entspricht, so ergibt sich eine Stagnation.

Dargestellt am deutschen »Musterland «: Im Jahr 2000 wuchsen die Investitionen der nicht-finanziellen Unternehmen stark, ihr Finanzierungsdefizit betrug I37,I Milliarden $€$, ihm standen Überschüsse der Haushalte (75,4 Milliarden $€$ ), des Auslands (26,7 Milliarden $€$ ) und des Staates (27,I Milliarden $€$ ) gegenüber (der Rest entfällt auf den für die Saldendynamik nicht relevanten Finanzsektor). Bis zum Jahr 2005 ergaben sich dramatische Verschiebungen: Sinkende (Real-)Investitionen drehten den Saldo der Unternehmen in einen Überschuss von 4,I Milliarden $€$ (bereits 2002 und 2004 hatte der Unternehmenssektor - erstmals in der Nachkriegszeit - Überschüsse erzielt), gleichzeitig steigerten die Haushalte ihren Überschuss auf I36,6 Milliarden €. Obwohl das Ausland ein Defizit von 88,4 Milliarden $€$ machte (Leistungsbilanzüberschuss Deutschlands), »erlitt« der Staat ein (Budget-)Defizit in Höhe von 74,5 Milliarden $€$.

8 Die Zusammenhänge zwischen Zinssatz, Wachstumsrate, Realkapitalbildung, den sektoralen Gesamt- und Primärsalden und der Akkumulationsdynamik von Realvermögen und (negativem) Finanzvermögen wird untersucht in Schulmeister (1996). 
Abbildung II: Gesamtwirtschaftliche Finanzierungssalden in Deutschland (in Prozent des BIP)

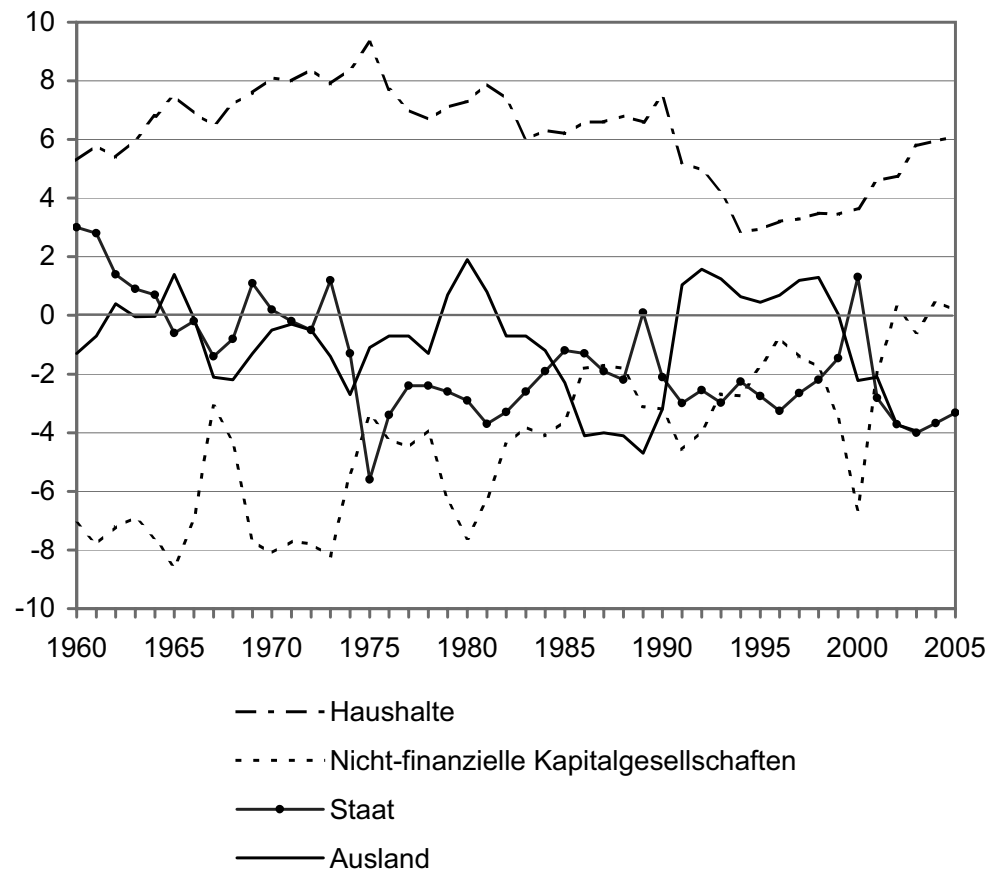

Quelle: Deutsche Bundesbank (Die Werte für die nicht-finanziellen Kapitalgesellschaften und den Staat im Jahr I995 wurden um den Sondereffekt der Auflösung der Treuhandanstalt bereinigt.)

Fazit: Die Finanzierungssalden in einer Volkswirtschaft sind »kommunizierende Gefäße«. Die traditionelle »Rolle« der Unternehmen ist es, die Überschüsse der Haushalte in Form von Investitionskrediten zu übernehmen und so in Realkapital und Arbeitsplätze zu verwandeln (wie in den I96oer Jahren; vgl. Abbildung II). Spielen die Unternehmer diese Rolle nicht mehr und erhöhen gleichzeitig auch die Haushalte ihr Sparen, dann ist eine nachhaltige Budgetkonsolidierung nicht möglich. Sparbemühungen verbessern unter diesen (finanzkapitalistischen) Bedingungen nicht den Budgetsaldo, sondern dämpfen das Wirtschaftswachstum. Dies kann zwar durch steigende Überschüsse in der Leistungsbilanz gemildert werden, auf Dauer lässt sich aber die Konsolidierungslast nicht an das Ausland abwälzen.

Auf dem Weg ans Ende der Sackgasse

Die bisher vorgestellten Beobachtungen und Beziehungen lassen sich in folgendem Bild zusammenführen:

- In einem globalen Kasino spielen etwa 20.000 professionelle Trader und zwei Millionen Amateure. Der Lauf der Kugel bestimmt die wichtigsten Preise in der Weltwirt- 
schaft: Wechselkurse, Rohstoffpreise, Aktienkurse und Zinssätze. Er folgt allerdings keinem Zufallsprozess, sondern hängt vom Spielverhalten der Teilnehmer ab. Wird mehr Kapital auf Rot (Kurssteigerung) gesetzt als auf Schwarz (Kursrückgang), so wird die Kugel mit größerer Wahrscheinlichkeit bei Rot landen.

- Die wichtigste systematische Komponente in der Entwicklung von Finanzmarktpreisen sind Kursschübe auf unterschiedlichen Zeitskalen. Sie akkumulieren sich zu mehrjährigen »bull markets" und »bear markets": die "manisch-depressiven " Schwankungen der wichtigsten »asset prices« um ihre theoretischen Gleichgewichtswerte.

- In ihrer Gesamtheit sind die Amateure die Verlierer im »Finanzkasino«, die professionellen (System-)Spieler die Gewinner.

- Auch die (großen) nicht-finanziellen Unternehmen haben immer mehr Kapital auf den Finanzmärkten (ein)gesetzt. Bei instabilen Wechselkursen, Rohstoffpreisen und Zinssätzen wurden diese Aktivitäten relativ zu Realinvestitionen zunehmend attraktiv. Dies dämpfte die Schaffung produktiver Arbeitsplätze und (damit) das Wirtschaftswachstum.

- Deutschland vollzog den Wechsel von einer real- zu einer finanzkapitalistischen »Wirtschaftskultur « besonders energisch. Die EUREX in Frankfurt wurde zur größten Derivatbörse der Welt und die Deutsche Bank zur zweitprofitabelsten "Spekulationsbank«. Gleichzeitig nahm die Arbeitslosigkeit besonders stark zu.

Darauf wurden die Unterstützungen für Arbeitslose gekürzt sowie die Ausgaben für Gesundheit und Pensionen. Die private Altersvorsorge wurde hingegen gefördert und damit auch der (neuerliche) Aktienboom. Fazit: Der private Konsum stagnierte, die Gesamtwirtschaft wuchs kaum, und auch das nur dank steigender Exporte.

Jeder Akteur folgt ökonomischer Rationalität: Die Unternehmer, welche die im Vergleich zur Realkapitalbildung höheren Finanzrenditen nutzen, die Finanzmathematiker, die optimale Spekulationsmodelle entwickeln, die Trader, welche solche "Spielsysteme« anwenden, die Finanzminister, welche im Sozialbereich sparen, um solide Staatsfinanzen zu gewährleisten, die verunsicherten Beschäftigten, welche aus Furcht unter ihren Verhältnissen leben, die Arbeitslosen, die sich an die Verschlechterung ihrer Lage anpassen müssen. Indes: Das Zusammenwirken dieser partiellen Rationalitäten hat ein irrationales Gesamtsystem hervorgebracht. An seinen »Früchten« lässt sich dies erkennen. Und an einem Vergleich mit den Systembedingungen in der ersten Hälfte der Nachkriegszeit:

- Das Gewinnstreben wurde systematisch auf realwirtschaftliche Aktivitäten gelenkt. Bei festen Wechselkursen, stabilen Rohstoffpreisen, niedrigen Zinssätzen und stagnierenden Aktienkursen war auf den Finanzmärkten nichts zu holen (vgl. Abbildungen 8 und I2).

- Der "Vermehrungsdrang« des Kapitals musste sich in der Realwirtschaft ausleben, Investitionen, Außenhandel und Gesamtproduktion wuchsen stark (vgl. Abbildungen Io und I2).

- Der Staat verbesserte die Wachstumsbedingungen durch Investitionen in die Infrastruktur und das Bildungswesen. 
Abbildung I2: Entwicklungstendenzen in den europäischen Industrieländern ${ }^{I}$
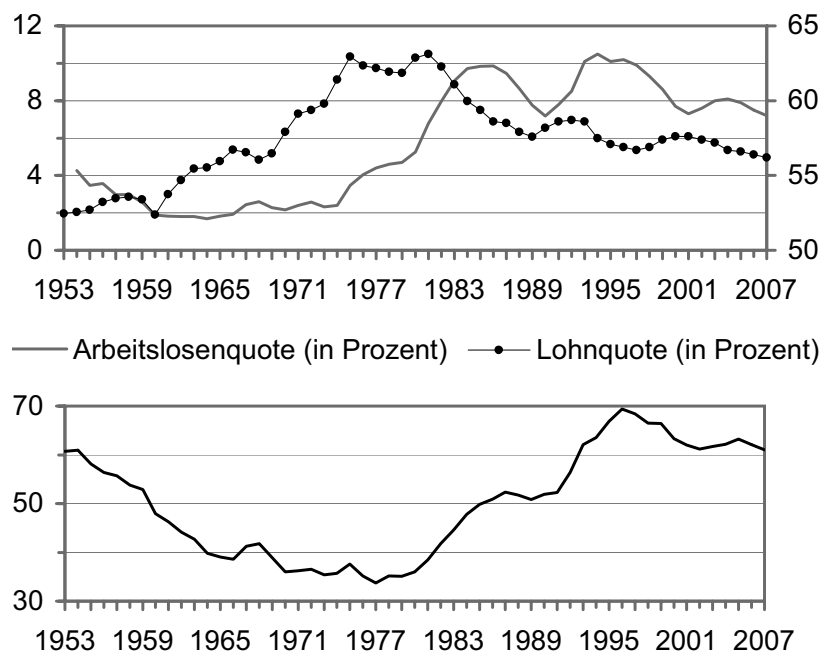

Staatsschuld (brutto in Prozent des BIP)

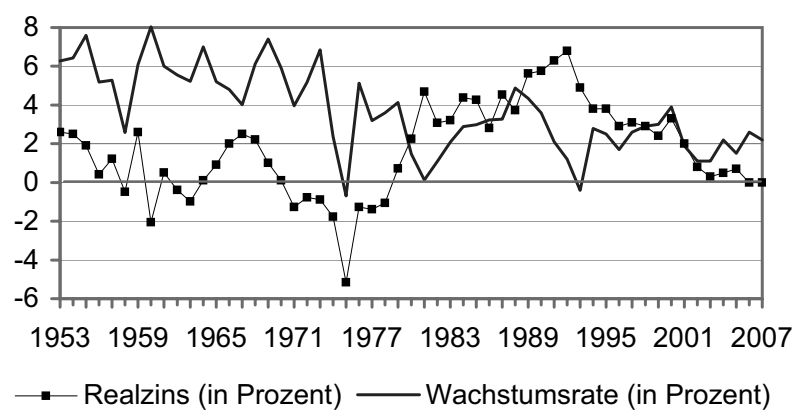

I Ab I99I: EU-I5, davor OECD-Europa (teilweise durch Daten für die drei größten Länder approximiert)

Quelle: OECD, Eurostat (Ameco)

- Das Vertrauen der Haushalte wurde durch den Ausbau der sozialen Sicherungssysteme gestärkt, der Konsum wuchs von Jahr zu Jahr.

- Im Hinblick auf die Finanzierungssalden galt: Die Überschüsse der Haushalte wurden durch Kreditaufnahme der Unternehmen in Realkapital und Arbeitsplätze transformiert, das Budget war annähernd ausgeglichen (vgl. Abbildung II).

- Bei Vollbeschäftigung ging die Staatsschuldenquote 20 Jahre lang zurück, gleichzeitig wurde der Sozialstaat mehr ausgebaut als je zuvor.

Die Eliten in Wissenschaft, Medien und Politik werden den irrationalen Charakter des finanzkapitalistischen Systems in seiner Gesamtheit noch einige Zeit nicht "wahr nehmen" (können). Täten sie es, sie würden den Glauben an die ökonomische Fundamentalwahrheit 
verlieren: Nur die Konkurrenz von Individuen auf freien Märkten ermöglicht das gesamtwirtschaftliche Optimum. Viel zuviel wurde in den letzten 30 Jahren in die Renovierung dieser Theorie des I9. Jahrhunderts investiert, als dass man sie rasch abschreiben könnte.

Allerdings: Der Holzwurm des Zweifels bohrt bereits seine Gedankengänge in ihrem Gebälk.

\section{Literatur}

Edwards, Franklin R. (1999): Hedge Funds and the Collapse of Long-Term Capital Management, in: The Journal of Economic Perspectives, Jg. I3, H. 2, S. I89-2IO

Friedman, Milton (1953), The Case for Flexible Exchange Rates in: Friedman, Milton ( $\mathrm{Hg}$.), Essays in Positive Economics, Chicago: University of Chicago Press, S. 157-203

Lowenstein, Roger (2000): When Genius Failed, New York: Random House

Lyons, Richard K. (1998): Profits and Position Control: A Week of FX Dealing, in: Journal of International Money and Finance, Jg. 17, H. I, S. 97-II5

Menkhoff, Lukas/Taylor, Mark P. (2007): The Obstinate Passion of Foreign Exchange Professionals: Technical Analysis, in: Journal of Economic Literature, im Erscheinen

Schulmeister, Stephan (1987): An Essay on Exchange Rate Dynamics, Wissenschaftszentrum Berlin, Berlin

Schulmeister, Stephan (1996): Zinssatz, Investitionsdynamik, Wachstumsrate und Staatsverschuldung, WIFO-Studie, Wien

Schulmeister, Stephan (I998): Die Beschäftigungsdynamik in den USA im Vergleich zu Deutschland und Japan, WIFO-Studie, Wien

Schulmeister, Stephan (2000): Globalization without Global Money: The Double Role of the Dollar as National Currency and as World Currency, in: Journal of Post Keynesian Economics, Jg. 22, H. 3, S. 365-395

Schulmeister, Stephan (2003): Aktienkursdynamik und Realkapitalbildung in den USA und Deutschland, WIFO-Studie, Wien

Schulmeister, Stephan (2004): Aktienkursdynamik und privater Konsum in den USA und Deutschland, WIFO-Studie, Wien

Schulmeister, Stephan (2005a): Purchasing Power Parities, Exchange Rates and International Price Competitiveness, WIFO-Studie, Wien

Schulmeister, Stephan (2005b): Die »ausgeblendeten« Ursachen der deutschen Wirtschaftskrise, in: Chaloupek, Günther/Heise, Arne/Matzner, Gabriele/Roth, Wolfgang (Hg.), Sisyphus als Optimist: Versuche zu zeitgemäßer politischer Ökonomie, Weiter-Denk-mal in Memoriam Egon Matzner, Hamburg, S. 94-II7

Schulmeister, Stephan (2006): The Interaction between Technical Currency Trading and Exchange Rate Fluctuations, in: Finance Research Letters, Jg. 3, H. 3, S. 2I2-233

The Economist (2006): Behind the Brass Plate, in: The Economist, 27.04.2006 
